\title{
Intelligent Model to Obtain Current Extinction Angle for a Single Phase Half Wave Controlled Rectifier with Resistive and Inductive Load
}

\author{
José Luis Calvo-Rolle ${ }^{1}$, Héctor Quintián², Emilio Corchado², Ramón \\ Ferreiro-García ${ }^{1}$
}

\begin{abstract}
The present work show the model of regression based on intelligent methods. It has been created to obtain current extinction angle for a half wave controlled rectifier. The system is a typically non-linear case of study that requires a hard work to solve it manually. First, all the work points are calculated for the operation range. Then with the dataset, to achieve the final solution, several methods of regression have been tested from traditional to intelligent types. The model is verified empirically with electronic circuit software simulation and analytical methods. The model allows obtaining good results in all the operating range.
\end{abstract}

Keywords: Half wave controlled rectifier, regression, non-linear model, MLP, SVM, polynomial models, LWP, K-NN.

\section{Introduction}

Electronic rectifiers are one of the most common circuits in electronic topics [1]. Its objective in general terms is to convert an alternate signal into continuous signal [2]. This type of circuits can be used in applications like DC power supplies [3], peak signal detectors [4], and so on.

Despite the simplest configuration of a rectifier circuit can be easy to understand and solve, for more complicate configurations they can be very difficult. Essentially, the difficulty depends of: the number of phases of the source, the characteristic of the load and if it is controlled or not controlled type [2].

The present research is focused on the single phase half wave controlled rectifier for resistive and inductive load. As would be seen in the case of study section, this variety of rectifier is not easy to solve in analytically form. The main reason is due to non-linear nature of this topology [3].

Usually to take a solution, to solve non-linear systems are used traditional methods with the help of computation tools. In this sense as methods to obtain a so-

\footnotetext{
${ }^{1}$ University of Coruña, Department of Industrial Engineering Avda. 19 de febrero, s/n, 15405, Ferrol, A Coruña, Spain \{jlcalvo, ferreiro\}@udc.es

${ }^{2}$ University of Salamanca, Departmento de Informática y Automática

Plaza de la Merced s/n, 37008, Salamanca, Spain \{escorchado, hector.quintian\} @usal.es
} 
lution can be cited: Trust-region dogled [5], Trust-region-reflective [6], Levenberg-Marquardt [7] [8] [9]. It is possible with these methods achieve good results in general terms with relatively few iterations.

Taking into account the fact that the operating range of the single phase half wave controlled rectifier for resistive and inductive load is known, it is possible to obtain the results of the angle extinction for the range. This fact is possible due the value do not depends the other parameters like voltage peak, frequency and so on. With the dataset is feasible apply regression and obtain automatically the extinction angle.

The traditional approaches for regression are based on Multiple Regression Analysis (MRA) methods [10]. MRA-based methods are very popular among others because have application in a lot of fields. It is well knowledge these methods have limitations [10] [11]. The limitations result from troubles associated with MRA-based methods, such as the inability of MRA to adequately deal with interactions between variables, nonlinearity, and multicollinearity [12] [10] [13]. More recently Softcomputing (SC)-based methods have been proposed as an option for many contributors as [11] [14] [15] [16] [17] [18].

Take into account all explained, a novel approach is proposed. The aim of the study is to develop an intelligent model that allows obtaining angle extinction of the single phase half wave controlled rectifier for resistive and inductive load. Many Softcomputing techniques have been tested in order to obtain the best fitness of the created model.

The work is structured as follow. It starts with a brief description of the single phase half wave controlled rectifier for resistive and inductive load. Afterwards intelligent regression methods tested in this study are described. Next section shows the model developed and the results for the different methods. Finally conclusions and future works are exposed.

\section{Case of study}

\subsection{Single phase half wave controlled rectifier for resistive and inductive load}

The single phase half wave controlled rectifier circuit with resistive and inductive load is showed in figure 1.

The load (resistance $R l$ and inductance $L 1$ ) is fed for the voltage source $V I$ via thyristor $X 1$. The principal characteristic of the circuit is that the current is not in phase with the voltage. This fact is due the inductive component of the load. The firing pulse is applied to the gate of thyristor $X 1$ (trigger pin) at instant defined as $\alpha$. It remains in the ON state until the load current tries to go to a negative value. This instant is defined as angle extinction $\beta$. The current $i(\omega t)$ is defined by equation 1, where $V m$ is the peak voltage, $\omega$ is the frequency, $t$ is the time, $\tau$ is $L / R$ and $\theta$ is tan $-1(\omega L / R)$.

$i(\omega t)=\frac{V_{m}}{Z}\left[\sin (\omega t-\theta)+\sin (\theta) \cdot e^{\frac{-\omega t}{\omega \tau}}\right]$ 


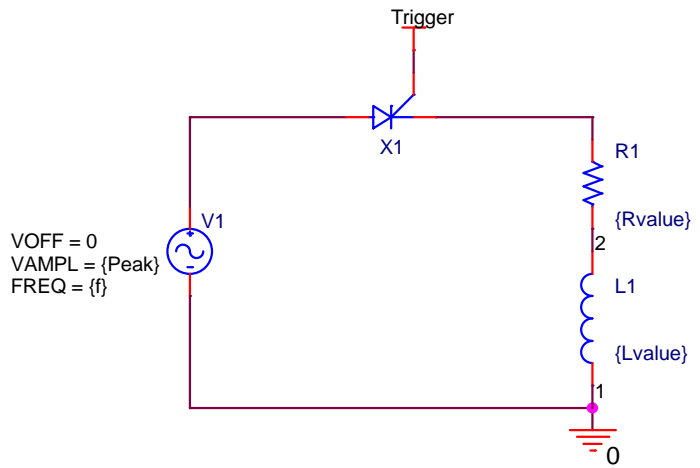

Fig. 1. Single phase half wave controlled rectifier for resistive and inductive load circuit.

Figure 2 show the voltage (dots in blue) and current in the load (continuous line in red). As can be shown, the voltage exists in the load when current exist. Angle extinction is specified at figure.

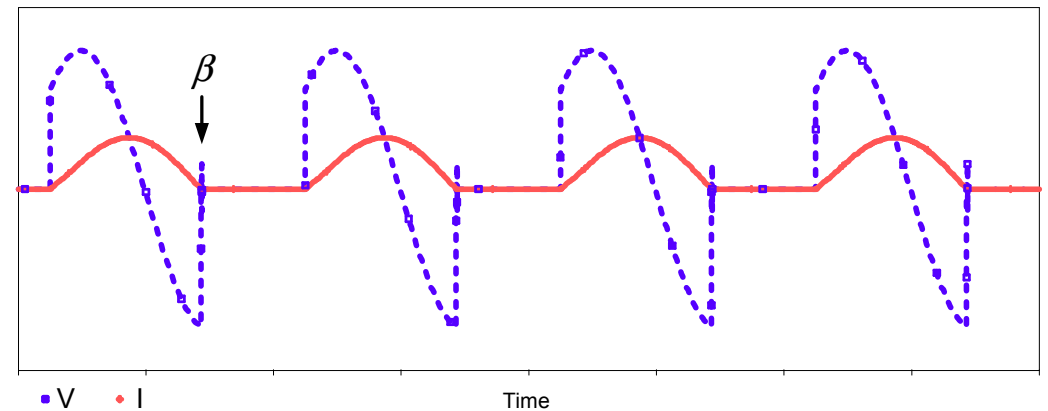

Fig. 2. Load Voltage and current.

To obtain angle extinction value $\beta$ it is necessary to solve the equation 2 [2].

$i(\beta)=\frac{V_{m}}{Z}\left[\sin (\beta-\theta)+\sin (\theta) \cdot e^{\frac{-\beta}{\omega \tau}}\right]=0$

There is not any analytical solution for the equation 2 , and it is necessary numeric methods to solve the equation like the mentioned above in the introduction.

\subsection{Novel approach}

The general schema of the proposed topology where intelligent model is used to obtain the value of angle extinction is illustrated in figure 3.

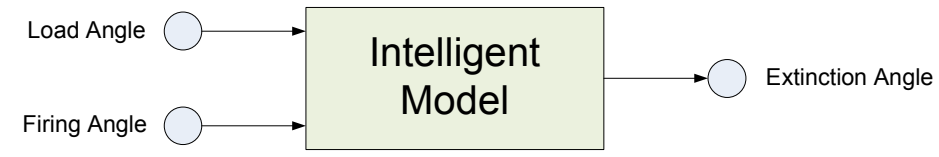

Fig. 3. General Schema 
As can be seen in the figure 2, the model has two inputs: the load angle and the firing angle. As an output, the model provide the extinction angle that the single phase half wave controlled rectifier circuit with resistive and inductive load has for the two mentioned parameters when it run.

\section{Model approach}

\subsection{Obtaining dataset}

For the dataset creation several things have been taken into account. Firstly it is necessary to include all the range, i.e. load angle should go from 0 degrees to 90 degrees. Know that this fact the combination of the values of the resistance and the inductance to obtain load angle must be adequate. At borderline values have been taken into account have been consider ideal values to complete the dataset.

In general terms the values of electric components have been chosen according with typical real values used at electric and electronic industry.

\subsection{Dataset conditioning}

For obtaining the required model, it has been used a dataset which has 2 input variables (alpha and fi) and 1 output variable (beta). The dataset with 3626 samples, has been divided in two dataset, training dataset (2417 samples, two thirds of the original dataset) and test dataset (1209, one third of the original dataset).

Each model has been trained and later evaluated with the test dataset, in terms of the MSE (Mean Square Error). In all techniques has been trained using cross validation with 10 folds.

\subsection{Used techniques}

Following each of the used techniques and its parameters are described.

Artifical Neural Networks (ANN). Multilayer Perceptron (MLP): A multilayer perceptron is a feed forward artificial neural network [19] [27]. It is one of the most typical ANNs due to its robustness and relatively simple structure. However the ANN architecture must be well selected to obtain good results. Tests were performed using 1 hidden layer, from 10 to 30 neurons in the hidden layer and log sigmoid and tangent sigmoid activation functions.

Finally the best results were obtained using 20 neurons and log sigmoidal transfer function for the hidden layer.

Support Vector Regression (SVR). Support Vector Regression (SVR) is a modification of the algorithm of the Support Vector Machines (SVM) [20] for classification. In SVR the basic idea is to map the data into a high-dimensional feature space $\mathrm{F}$ via a nonlinear mapping and to do linear regression in this space.

LS-SVM. Least Square Support Vector Machine (LS-SVM). Least Square formulation of SVM, are called LS-SVM, in the approximation the solution is ob- 
tained by solving a system of linear equations, and it is comparable to SVM in terms of generalization performance [21]. The application of LS-SVM to regression is known as LS-SVR (Least Square Support Vector Regression). In LS-SVR, the $\varepsilon$-insensitive loss function is replaced by a classical squared loss function, which constructs the Lagrangian by solving the linear Karush-Kuhn-Tucker (KKT) system:

$\left[\begin{array}{cc}0 & I_{n}^{T} \\ I_{n} & K+\gamma_{-1} I\end{array}\right]\left[\begin{array}{c}b_{0} \\ b\end{array}\right]=\left[\begin{array}{l}0 \\ y\end{array}\right]$

Where $I_{n}$ is a [nx1] vector of ones, T means transpose of a matrix or vector, $\gamma$ a weight vector, $b$ regression vector and $b_{0}$ is the model offset. In LS-SVR, only 2 parameters $(\gamma, \sigma)$ are needed. Where $\sigma$ is used the width of the kernel [22].

LS-SVM [23] Matlab toolbox has been used. In this toolbox, the tuning of the parameters $(\gamma, \sigma$ equation 3$)$ is conducted in two steps. First, a state-of-the-art global optimization technique, Coupled Simulated Annealing (CSA) [24], determines suitable parameters according to some criterion. Second, these parameters are then given to a second optimization procedure (simplex or gridsearch) to perform a fine-tuning step.

The optimal parameters obtained by the previous method are: $\gamma=$ $2.1515 e^{7}, \sigma^{2}=1.99477$

Polynomial models. Generally, a polynomial regression model may also be defined as a linear summation of basis functions:

$F(x)=\sum_{i=1}^{k} a_{i} f_{i}(x)$

where $k$ is the number of basis functions (equal to the number of model's parameters); $f_{i}(x)(I=1,2, \ldots, k)$ is a predefined polynomial basis function. The number of the basis functions in polynomial model of degree $p$ is:

$m=\prod_{i=1}^{p}(1+d / i)$

The estimation of model's parameters is made based on the training data typically using the Ordinary Least-Squares (OLS) method, minimizing:

$a=\arg \min _{a} \sum_{i=1}^{n}\left(y_{(i)}-F\left(x_{(i)}\right)\right)^{2}$

where $\mathrm{x}_{(i)}$ is the vector of input variables' values of the $i_{\text {th }}$ data point and $\mathrm{y}_{(i)}$ is the output value of that point. In this polynomials method as well as all the other regression modeling methods, the systems of linear equations in OLS are solved using Gaussian elimination and back substitution.

The best approximation has been obtained using Degree $=5$.

Locally Weighted Polynomials (LWP). Locally Weighted Polynomial approximation [25] is designed to address situations in which the models of global behaviour do not perform well or cannot be effectively applied without undue effort. The LWP approximation is carried out by point wise fitting of low-degree polynomials to localized subsets of the data.

The coefficients of the polynomial are calculated, minimizing: 
$a=\arg \min _{a} \sum_{i=1}^{n} w\left(x_{\text {query }}, x_{(i)}\right)\left(F\left(x_{(i)}-y_{(i)}\right)\right)^{2}$

where $w$ is a weight function; $x_{\text {query }}$ is the query point nearest neighbours of which will get the largest weights. The weight function $w$ depends on the Euclidean distance (in scaled space) between the point of interest $x_{\text {query }}$ and the points of observations $x$. The kind of weighting for Gaussian weight function implemented is:

$w\left(x_{\text {query }}, x_{(i)}\right)=\exp \left(-\alpha \mu_{(i)}^{2}\right)$

where $\alpha$ is a coefficient (bandwidth is $\mathrm{n} \alpha$, where $\mathrm{n}$ is the number of total points) and the $\mu_{(i)}$ is a scaled distance from the query point to the $\mathrm{i}_{\text {th }}$ point in the training data set:

$\mu_{i}=\frac{\left\|x_{\text {query }}-x_{(i)}\right\|}{\left\|x_{\text {query }}, x_{\text {farthest }}\right\|}$

where $\|\cdot\|$ is the Euclidean norm; $x_{\text {farthest }}$ is the farthest training point from the point $x_{\text {query }}$. The locality of the approximation is controlled by varying the value of the coefficient $\alpha$. If $\alpha$ is equal to zero then local approximation transforms into global approximation.

The best approximation has been obtained using degree 6 and bandwidth 58.75.

K-Nearest Neighbours (K-NN). K-NN technique [26] is a nonparametric method, which generally, can be written as:

$F(x)=\frac{\sum_{i=1}^{k} w_{i} y_{i}^{\prime}}{\sum_{i=1}^{k} w_{i}}$

where $y_{i}^{\prime}$ is the output value of $i_{\text {th }}$ point from the $k$ points nearest to the query point $x$ and $\mathrm{w}_{\mathrm{i}}$ is the weight of that point. Here closeness implies the Euclidean distance. In this case the distance weighting schemes used is:

$w_{i}=1-\left\|x-x_{i}^{\prime}\right\|(i=1,2, \ldots, k)$

where $x_{(i)}^{\prime}$ is the input value of $i_{\text {th }}$ point from the nearest ones.

The best approximation has been obtained using number of nearest neighbours $=6$.

\section{Results}

Once the models were trained, they were tested getting the following results in terms of MSE:

With showed results, it is possible to conclude that the best models are ANN and SMV-LS. They are more than 10 times better than the other tested methods. ANN and SVM-LS obtain similar results, but the computational cost required for training process of SVM-LS is so much higher than ANN. The main reason is due to the required optimization process of SVM-LS for getting the best parameters $(\gamma, \sigma)$ necessary for the training process, but once it has been trained, the computational cost is the same as ANN. 
Table 1. Results for each of the applied methods.

\begin{tabular}{lll}
\hline Method & Parameters & MSE (Test dataset) \\
\hline ANN & $\begin{array}{l}\text { 1 hidden layer, 20 neurons, log sigmoidal ac- } \\
\text { tivation function }\end{array}$ & 0.000031147 \\
SVM & $\gamma=2.1515 \mathrm{e}^{7}, \sigma^{2}=1.99477$ & 0.000031634 \\
Polynomial & Degree $=5$ & 0.033615344 \\
LWP & Degree 6 and bandwidth 58.75 & 0.00022042078 \\
KNN & Number of nearest neighbours $=6$ & 0.49652762 \\
\hline
\end{tabular}

\section{Conclusions}

With the novel approach to obtain the extinction angle, as a principal conclusion, put in highlight that very good results have been achieved in general terms. With the intelligent model created based on Soft computing techniques is not necessary to solve the non-linear equation well analytically or by simulation.

As can be seen in results section the best approximation is obtained with ANN, where the MSE has a value minor than 0.000032. Other techniques allow good results like SVM or LWP. With any of them, the angle extinction would be calculated, among others because the real components have tolerances around $5 \%$.

\section{References}

1. Malvino, A.P., Bates, D.J.: Electronic principles. Princeton, NJ, Recording for Blind \& Dyslexic (2008)

2. Hart, D.W.: Power Electronics. McGraw-Hill, New York (2011)

3. Rashid, M H: Power electronics handbook: devices, circuits, and applications. Burlington, MA: Butterworth-Heinemann (2011)

4. Mohan, N.: Power electronics: a first course. Hoboken, N.J (2012)

5. Coleman, T.F., Li, Y.: An Interior, Trust Region Approach for Nonlinear Minimization Subject to Bounds. SIAM Journal on Optimization 6, 418-445 (1996)

6. Coleman, T.F., Li, Y.: On the Convergence of Reflective Newton Methods for Large-Scale Nonlinear Minimization Subject to Bounds. Mathematical Programming 67(2), 189-224 (1994)

7. Levenberg, K.: A Method for the Solution of Certain Problems in Least-Squares. Quarterly Applied Mathematics 2, 164-168 (1944).

8. Marquardt, D.: An Algorithm for Least-squares Estimation of Nonlinear Parameters. SIAM Journal Applied Mathematics 11, 431-441 (1963)

9. Moré, J. J.: The Levenberg-Marquardt Algorithm: Implementation and Theory. Numerical Analysis, ed. G. A. Watson, Lecture Notes in Mathematics 630, Springer Verlag, 105-116 (1977)

10. Mark, J., Goldberg, M.: Multiple Regression Analysis and Mass Assessment: A Review of the Issues. Appraisal Journal 56(1), 89-109 (1988)

11. Do, A.Q., Grudnitski, G.: A Neural Network Approach to Residential Property Appraisal. The Real Estate Appraiser 58(3), 38-45 (1992)

12. Larsen, J.E., Peterson, M.O.: Correcting for Errors in Statistical Appraisal Equations. The Real Estate Appraiser and Analyst 54(3), 45-49 (1988)

13. Limsombunchai, V., Gan, C., Lee, M.: House Price Prediction: Hedonic Price Model Vs. Artificial Neural Network. American Journal of Applied Sciences 1(3), 193-201 (2004)

14. Worzala, E., Lenk, M., Silva, A.: An Exploration of Neural Networks and Its Application to Real Estate Valuation. Journal of Real Estate Research 10, 185-202 (1995) 
15. Guan, J., Levitan, A.S.: Artificial Neural Network Based Assessment of Residential Real Estate Property Prices: A Case Study. Accounting Forum 20(3/4), 311-26 (1997)

16. Taffese, W.Z.: Case-Based Reasoning and Neural Networks for Real Estate Valuation. Proceedings of 25th International Multi-Conference: Artificial Intelligence and Applications, 84-9, Innsbruck, Austria (2007)

17. Guan, J., Zurada, J., Levitan, A.S.: An Adaptive Neuro-Fuzzy Inference System Based Approach to Real Estate Property Assessment. Journal of Real Estate Research 30(4), 395-420 (2008)

18. Peterson, S., Flanagan, A.B.: Neural Network Hedonic Pricing Models in Mass Real Estate Appraisal. Journal of Real Estate Research 31(2), 147-64 (2009)

19. Bishop, C.M.: Pattern recognition and machine learning. Springer, New York (2006)

20. Cristianini, N., Shawe-Taylor, J.: An Introduction to Support Vector Machines. Cambridge University Press, Cambridge (2000)

21. Ye, J., Xiong, T.: Svm versus least squares svm. The 11th International Conference on Artificial Intelligence and Statistics (AISTATS), 640-647 (2007)

22. Yankun, L., Xueguang, S., Wensheng, C.: A consensus least support vector regression (LSSVR) for analysis of near-infrared spectra of plant samples. Talanta 72, 217-222 (2007)

23. De Brabanter, K., Karsmakers, P., Ojeda, F., Alzate, C., De Brabanter, J., Pelckmans, K., De Moor, B., Vandewalle, J., Suykens, J.A.K.: LS-SVMlab Toolbox User's Guide version 1.7 (2010). http://www.esat.kuleuven.be/sista/lssvmlab/

24. Xavier de Souza, S., Suykens, J.A.K., Vandewalle, J., Bolle, D.: Coupled Simulated Annealing, IEEE Transactions on Systems, Man and Cybernetics - Part B, 40(2), 320-335 (2010).

25. Cleveland W. S., Devlin S. J.: Locally weighted regression: An approach to regression analysis by local fitting, Journal of the American Statistical Association 83, 596-610 (1988)

26. Duda, R.O., Hart, P.E., Strork, D.G.: Pattern Classification, $2^{\text {nd }}$ ed. Wiley, Chichester (2001)

27. David, J., Henao, V.: Neuroscheme: A modeling language for artificial neural networks. Dyna-Colombia, Vol 147: 75-82 (2005) 